\title{
COVID-19 and the Mental Health of Frontline Nurses: A Cross-Sectional Study in Southwestern Iran
}

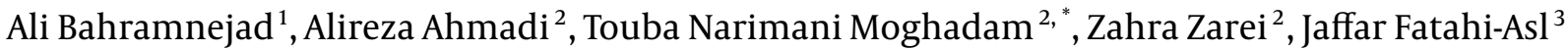 \\ and Ghasem Yadegarfar ${ }^{4}$ \\ ${ }^{1}$ Neuroscience Research Center, Institute of Neuropharmacology, Kerman University of Medical Sciences, Kerman, Iran \\ ${ }^{2}$ Behbahan Faculty of Medical Sciences, Behbahan, Iran \\ ${ }^{3}$ Department of Radiology Technology, School of Allied Medical Sciences, Ahvaz Jundishapur University of Medical Sciences, Ahvaz, Iran \\ ${ }^{4}$ Department of Statistics \& Epidemiology, School of Health, Isfahan University of Medical Sciences, Isfahan, Iran \\ "Corresponding author: Behbahan Faculty of Medical Sciences, Behbahan, Iran. Email: narimani20167@gmail.com
}

Received 2021 May 24; Revised 2021 October 03; Accepted 2021 November 05.

\begin{abstract}
Background: Nurses, as frontline health workers, are exposed to the risks of adverse mental health outcomes due to their direct contact with COVID-19 patients.

Objectives: The present study aimed to investigate the mental health of frontline nurses in Behbahan, southwestern Iran.

Methods: In this web-based cross-sectional study, data from 173 frontline nurses were collected in 3 COVID-19 referral hospitals in Behbahan, southwestern Iran. This study included demographic information and General Health Questionnaire (GHQ-28). Logistic regression analysis was used to examine the variables affecting mental health.

Results: The present study included 173 frontline nurses (nurses and nurse assistants), $71.7 \%$ of whom were female and $28.3 \%$ of whom were male. Further, $76.3 \%$ of frontline nurses were suspected of poor mental health, and $23.7 \%$ were mentally healthy. The results of the multivariable logistic regression model showed that female participants had poorer mental health than men (odds ratio $[\mathrm{OR}], 2.51 ; 95 \% \mathrm{CI}, 1.16-5.41 ; \mathrm{P}=0.01$ ). The mental health of nurses with a history of COVID-19 infection was poorer than that of nurses without a history of COVID-19 infection (OR, 3.3; 95\% CI, 1.42 - 7.53; P = 0.006).

Conclusions: The findings of our study showed that female nurses with a history of COVID-19 were more at risk for poor mental health. These groups need more attention and support in epidemics.
\end{abstract}

Keywords: Iran, COVID-19, Mental Health, Nurses

\section{Background}

The international community is concerned about COVID-19 (Coronavirus disease 2019) and its long-term consequences. Different areas of life, such as global markets, industries, agriculture, economy, human health, health care, etc, are being affected by COVID-19 (1). In the meantime, we have witnessed many psychosomatic and psychological problems among different groups of people, COVID-19 patients, and health care workers (2). In this regard, the World Health Organization (WHO) and International Mental Health Associations have warned that the current COVID-19 pandemic will lead to a severe increase of stress-related conditions and mental health issues worldwide (3).

Anxiety, uncertainty, feelings of fear, loneliness, stress, and depression have been reported in the general population worldwide after the outbreak of COVID-19 (4); on the other hand, emerging reports have been documented an increase in anxiety-related symptoms, stress, and depression, particularly among vulnerable people (i.e., economically/socially disadvantaged people and chronically/mentally ill populations) (5, 6). Even among suspected patients and those with COVID-19, in addition to the physical pain, they suffer from mental pressure (such as depression, anxiety, insomnia, and hopelessness), which can reduce adherence to treatment and might invoke aggression and suicide (7). The outbreak of the COVID-19 pandemic has increased concerns about the mental health of Iranians. In this regard, the prevalence and severity of psychiatric disorders in COVID-19 patients have been widely studied (8). Meanwhile, health professionals are no exception to this rule, and during an epidemic, they are more at risk of adverse mental health outcomes (7, 9-11).

Among health professionals, nurses, as frontline health workers, are exposed to many risks, including 
occupational hazards and mental pressures, due to their direct contact with COVID-19 patients. Even nurses' past experiences in dealing with SARS indicate many problems, including feelings of anger, guilt, fear of death, loneliness, physical disorders, uncertainty, and mismanagement of information (12). The results of a study in Iran showed that nurses who cared for COVID-19 patients experienced many psychological problems (11). A recent study in China on 1257 frontline health workers showed that nurses were at greater risk of depression, anxiety, distress, and insomnia than other health professionals (13). The results of another study in Spain showed that more than half of the nurses had poor mental health, which was higher than other health workers (14). Due to the seriousness of COVID-19 and prolonged exposure with patients, mental challenges among nurses can affect their physical and mental health and reduce their efficiency (15).

\section{Objectives}

The present study was aimed to investigate the mental health of frontline nurses in Behbahan, southwestern Iran, using the General Health Questionnaire (GHQ-28).

\section{Methods}

In this web-based cross-sectional study, 173 frontline nurses (nurses and nurse assistants) working in COVID19 referral hospitals (ie, Shahidzadeh, Farideh Behbahani, and Shahid Mostafa Khomeini hospitals in Behbahan)were studied to investigate mental health, and factors affecting them.

Inclusion criteria included frontline nurses (nurses and nurse assistants) working in the above 3 hospitals, age over 18 years, and signed informed consent to participate in the study.

Finally, out of 180 nurses who filled in the questionnaire, 7 questionnaires were excluded from the study due to incomplete completion of the questionnaire (failure to complete more than one-third of the questionnaire questions), and a total of 173 nurses who met the inclusion criteria were included in the study in an accessible manner. In this study, to collect the data, a 2-part questionnaire (including demographic information and GHQ-28) was used and shared from November 20 to December 10, 2020, with the cooperation of all 3 hospitals among the nurses of COVID-19 wards. Demographic information in this questionnaire included age, gender, level of education, marital status, and work experience. Participants were also asked about their history of COVID-19 infection (yes, no); this information was recorded as a variable in the research.
GHQ-28 is a popular self-assessment questionnaire employed to monitor and screen people at risk for psychiatric disorders. This questionnaire consists of 28 items and 4 scales, which each scale contains 7 items. Items can be categorized as follows: (1) items 1 - 7: physical symptoms; (2) items 8 - 14: anxiety and insomnia symptoms; (3) items 15 - 21: social functioning; and (4) items 22 - 28: depression. The scoring of this questionnaire is based on a Likert scale [never (0), sometimes (1), most of the time (2), almost always (3)]. The total score of the 4 subscales ranges from 0 to 84 . The cut point for the test is considered to be 23 . In other words, the study group was categorized into with and without psychological distress using a GHQ-28 cut-off of 23 ( $\leq 23$ : without a mental disorder, $>23$ : with a mental disorder) (16-19). The validity and reliability of this questionnaire have already been confirmed in Iran (20-22). In addition, in the current study, the reliability of this questionnaire was approved by the Cronbach $\alpha(0.81)$.

This study was approved by the Ethics Committee of Behbahan Faculty of Medical Sciences, Behbahan, Iran (code: IR.BHN.REC.1399.022).

\subsection{Statistical Analysis}

Categorical variables were summarized as counts and percentages. The $\chi^{2}$ test was used to test the differences among the groups. We used univariate and multivariate logistic regression methods to adjust for the effects of independent variables. First, all independent variables were entered into the univariate logistic regression model, and variables with a P value of less than 0.2 were entered into the multivariable logistic regression model. The final model was achieved using a backward selection approach. A P value of less than 0.05 was considered significant. All analyses in this study were performed using SPSS version 26 (SPSS Inc, Chicago, Ill, USA).

\section{Results}

The present study included 173 frontline nurses (nurses and nurse assistants), most of whom (124) were women (71.7\%), and $112(64.7 \%)$ of the participants were married. The data also showed that 132 (76.3\%) had a bachelor's degree, $66(38.2 \%)$ had work experience of less than 5 years, and 79 (38.5\%) had a history of COVID-19 infection. A total of 76.3\% of frontline nurses were suspected of poor mental health. We used the chi-square test to compare the differences between the groups for mental health (score $\leq 23$, score $>23$ ). In 2 groups related to mental health, there was a significant difference between gender, level of education, and history of COVID-19 infection (Table 1).

The mean general health score was $9.49 \pm 31.56$. Among the 4 GHQ-28 scales, the higher and lower scores 


\begin{tabular}{|c|c|c|c|c|}
\hline \multirow{2}{*}{ Variables } & \multicolumn{4}{|c|}{ Mental Health } \\
\hline & No. (\%) & $\begin{array}{l}\text { With a Mental Disorder } \\
\text { Score }>23\end{array}$ & $\begin{array}{c}\text { Without a Mental } \\
\text { Disorder Score } \leq 23\end{array}$ & P Value \\
\hline Overall & 173 & $132(76.3)$ & $41(23.7)$ & - \\
\hline Age & & & & 0.431 \\
\hline $18-25$ & $18(10.4)$ & $15(83.3)$ & $3(16.7)$ & \\
\hline $26-35$ & $92(53.2)$ & $70(76.1)$ & $22(23.9)$ & \\
\hline $36-45$ & $51(29.5)$ & $40(78.3)$ & $11(21.7)$ & \\
\hline $46-80$ & $12(6.9)$ & $7(58.3)$ & $5(41.7)$ & \\
\hline Gender & & & & 0.037 \\
\hline Male & $49(28.3)$ & $32(65.3)$ & $17(34.7)$ & \\
\hline Female & $124(71.7)$ & $100(80.6)$ & $24(19.4)$ & \\
\hline Marital status & & & & 0.369 \\
\hline Single & $61(35.3)$ & $49(80.3)$ & $12(19.7)$ & \\
\hline Married & $112(64.7)$ & $83(74.1)$ & $29(25.9)$ & \\
\hline Education & & & & 0.04 \\
\hline Associate degree & $30(17.3)$ & $25(83.3)$ & $5(16.7)$ & \\
\hline Bachelor's degree & $132(76.3)$ & $102(77.3)$ & $3022.7)$ & \\
\hline Master's degree/ doctorate & $11(6.4)$ & $5(45.5)$ & $6(54.5)$ & \\
\hline Work experience & & & & 0.75 \\
\hline Less than 5 years & $66(38.2)$ & $50(75.8)$ & $16(24.2)$ & \\
\hline 5 to 10 years & $41(23.7)$ & $33(80.1)$ & $8(19.1)$ & \\
\hline More than 10 years & $66(38.2)$ & $49(74.2)$ & $17(25.8)$ & \\
\hline History of COVID-19 infection & & & & 0.007 \\
\hline Yes & $69(38.5)$ & $60(86.9)$ & $9(13.1)$ & \\
\hline No & $104(61.5)$ & $72(69.2)$ & $72(20.8)$ & \\
\hline
\end{tabular}

were related to social dysfunction and depression, respectively (Table 2).

In the univariate model, variables of gender, level of education, and history of COVID-19 infection were among the factors associated with patients' mental health $(\mathrm{P}<$ 0.2 ). The results of the multivariable logistic regression model showed that the variables of gender and history of infection were significant $(\mathrm{P}<0.05)$. Additionally, female nurses had poorer mental health than male nurses (OR, 2.51; 95\% CI, 1.16 - 5.41; $\mathrm{P}=0.012$ ), and the mental health of nurses with COVID-19 was lower than that of nurses without COVID-19 (OR, 3.3; 95\% CI, 1.42 - 7.53; P = 0.006; Table 3).

\section{Discussion}

The findings of the present study showed that $76.3 \%$ of frontline nurses were suspected of poor mental health; this rate was significantly higher in our study than in previous mental health surveys done on Iranian nurses under non-COVID-19 conditions (in the study by Dehvan et al, it was $57.35 \%$, and in the study by Taghinejad et al, it was $43.2 \%$ ) $(23,24)$.

Nurses are at risk of infection due to close contact with patients and their families. On the other hand, the high risk of transmitting this disease to their own families and relatives causes great physical stress and emotional and mental pressures $(25,26)$. The public health crisis of COVID19 or SARS has had a serious impact on health workers, especially frontline nurses, because the frontline nurses are the main health workers in dealing with public health emergencies, and their psychological well-being is vital to overcoming critical health conditions (27).

Even previous studies on nurses who provided care for SARS patients have shown that they are at greater risk of mental disorders, including anxiety, depression, panic, dis- 


\begin{tabular}{|c|c|c|c|c|}
\hline \multirow{2}{*}{$\begin{array}{l}\text { Scores for GHQ-28 and its subscales } \\
\text { Somatic symptoms }\end{array}$} & \multirow{2}{*}{$\begin{array}{l}\text { Mean } \pm \text { SD } \\
8.05 \pm 3.85\end{array}$} & Minimum & \multicolumn{2}{|r|}{ Maximum } \\
\hline & & 1 & \multicolumn{2}{|r|}{19} \\
\hline Anxiety/insomnia & $7.68 \pm 5.03$ & \multicolumn{2}{|c|}{0} & 21 \\
\hline Social dysfunction & $12.29 \pm 2.92$ & \multicolumn{2}{|c|}{4} & 20 \\
\hline Severe depression & $3.55 \pm 4.12$ & \multicolumn{2}{|c|}{0} & 18 \\
\hline GHQ-28 total score & $31.56 \pm 9.49$ & \multicolumn{2}{|c|}{15} & 59 \\
\hline \multicolumn{5}{|l|}{ Mental Health } \\
\hline \multirow{2}{*}{ Variables } & \multicolumn{2}{|c|}{ Univariate } & \multicolumn{2}{|c|}{ Multivariable } \\
\hline & Odds Ratio (95\% CI) & P Value & Odds Ratio (95\% CI) & P Value \\
\hline \multicolumn{5}{|l|}{ Age } \\
\hline $18-25$ & $3.6(0.66-7.34)$ & 0.141 & - & - \\
\hline $26-35$ & $2.3(0.66-5.33)$ & 0.210 & - & - \\
\hline $36-45$ & $2.6(0.69-9.6 .2)$ & 0.163 & - & - \\
\hline $46-80$ & 1 & 1 & - & - \\
\hline \multicolumn{5}{|l|}{ Gender } \\
\hline Female & $2.21(1.06-4.63)$ & 0.033 & $2.51(1.16-5.41)$ & 0.011 \\
\hline Male & 1 & 1 & 1 & 1 \\
\hline \multicolumn{5}{|l|}{ Marital status } \\
\hline Single & $1.42(0.67-3.1)$ & 0.365 & - & - \\
\hline Married & 1 & 1 & - & - \\
\hline \multicolumn{5}{|l|}{ Education } \\
\hline Associate degree & $2.21(1.3-9.24)$ & 0.042 & - & - \\
\hline Bachelor's degree & $1.89(1.2-7.52)$ & & - & - \\
\hline Master's degree/doctorate & 1 & 1 & - & - \\
\hline \multicolumn{5}{|l|}{ Work experience } \\
\hline Less than 5 years & $1.08(0.493-2.38)$ & 0.756 & - & - \\
\hline 5 to 10 years & $1.43(0.55-3.7)$ & & - & - \\
\hline More than 10 years & 1 & 1 & - & - \\
\hline \multicolumn{5}{|l|}{ History of COVID-19 infection } \\
\hline Yes & $2.96(1.3-6.69)$ & 0.009 & $3.3(1.42-7.53)$ & 0.006 \\
\hline No & 1 & 1 & 1 & 1 \\
\hline
\end{tabular}

trust, delirium, post-traumatic stress, and even suicidal thoughts (28). A recent study in Oman on health workers showed that the COVID-19 outbreak had a significant impact on the mental health of health workers, including nurses; accordingly, frontline nurses and health workers were most affected by the global health crisis (29). Further, another study in China found that frontline nurses in COVID-19 wards had a higher rate of mental health problems.
Health professionals play an important role in managing a pandemic, especially nurses, as the biggest group of health workers who spend more time with patients than other health professionals and have an important role in the management and treatment of patients. According to the ethical reciprocity principle, hospitals have a reciprocal duty to health care staff, such as providing health facilities when caring for patients with a new illness, communicating with them to control infection, and providing per- 
sonal protective equipment and psychological screening, as well as motivational and mental support and psychiatric counseling. As a result, psychological needs, especially in nurses who provide care for COVID-19 patients, require immediate attention (28).

The findings of the present study showed that female nurses had poorer mental health than male nurses. The results of a study in Pakistan on female health workers (doctors, nurses, etc.) showed that they were not eligible for quarantine after work and contact with COVID-19 patients due to their family and social responsibilities. This led to fear of virus transmission, severe frustration, and conflict between work and family commitment (18). The findings of other studies on frontline nurses in Iran, China, and Italy were also consistent with the results of the present study $(11,13,30)$.

We further found that nurses with COVID-19 had poorer mental health than other nurses. In fact, nurses with COVID-19 may experience psychological problems due to fear of transmitting the virus to colleagues, patients, and family members (31).

\subsection{Limitations and Strengths}

Our study has some limitations. First, we only focused on frontline nurses, and it was conducted only in Behbahan, so the findings cannot be generalized to the entire nursing community. Also, conducting an online survey and limited observation on the eligibility of participants and self-report data gathering were other limitations of the study.

However, our study has some strengths. The major strength of this study was the description, evaluation, and recognition of factors affecting the mental health and stress levels of frontline nurses. In this study, using logistic regression, we found valuable new findings regarding the mental health of frontline nurses. Among them, we can mention the poorer mental health of female nurses than male nurses. Given the current situation and the high prevalence of COVID-19, these results can help policymakers to implement appropriate interventions to reduce the psychological effects of COVID-19 on nurses.

\subsection{Conclusion}

The findings of this study showed that a high percentage of frontline nurses were Suspected of poor mental health. Further, female nurses had poorer mental health than male nurses, and nurses with COVID-19 were at higher risk for poor mental health than nurses without COVID-19. In such a critical situation, it is better to pay more attention to this group of nurses, especially female nurses.

\section{Acknowledgments}

This study was supported by Behbahan Faculty of Medical Sciences.

\section{Footnotes}

Authors' Contribution: Study concept and design, T.N. M, and A. B.; Acquisition of data, A. A., and Z.Z.; Analysis and interpretation of data, T.N. M under the supervision GH. Y., and J. F. A; Drafting of the manuscript, T.N. M under the supervision A.B., and J. F A.; Statistical analysis, T.N. M, and GH. Y.; Study supervision, J. F. A and A. B.

Conflict of Interests: The authors declare no conflict of interests.

Ethical Approval: This study was approved by the Ethics Committee of Behbahan Faculty of Medical Sciences (code: IR.BHN.REC.1399.022; http://ethics.research.ac.ir/IR.BHN.REC.1399.022).

Funding/Support: This study was supported by Behbahan Faculty of Medical Sciences.

\section{References}

1. Kumar A, Nayar KR. COVID 19 and its mental health consequences. J Ment Health. 2021;30(1):1-2. doi: 10.1080/09638237.2020.1757052. [PubMed: 32339041].

2. Pfefferbaum B, North CS. Mental Health and the Covid-19 Pandemic. $N$ Engl J Med. 2020;383(6):510-2. doi: 10.1056/NEJMp2008017. [PubMed: 32283003].

3. Kaufman KR, Petkova E, Bhui KS, Schulze TG. A global needs assessment in times of a global crisis: world psychiatry response to the COVID-19 pandemic. BJPsych Open. 2020;6(3). e48. doi: 10.1192/bjo.2020.25. [PubMed: 32250235]. [PubMed Central: PMC7211994].

4. Torales J, O'Higgins M, Castaldelli-Maia JM, Ventriglio A. The outbreak of COVID-19 coronavirus and its impact on global mental health. Int J Soc Psychiatry. 2020;66(4):317-20. doi: 10.1177/0020764020915212. [PubMed: 32233719].

5. Bao Y, Sun Y, Meng S, Shi J, Lu L. 2019-nCoV epidemic: address mental health care to empower society. Lancet. 2020;395(10224):e37-8. doi: 10.1016/S0140-6736(20)30309-3. [PubMed: 32043982]. [PubMed Central: PMC7133594]

6. Torales J, Rios-Gonzalez C, Barrios I, O'Higgins M, Gonzalez I, Garcia O, et al. Self-Perceived Stress During the Quarantine of COVID19 Pandemic in Paraguay: An Exploratory Survey. Front Psychiatry. 2020;11:558691. doi: 10.3389/fpsyt.2020.558691. [PubMed: 33192674]. [PubMed Central: PMC7649175].

7. Li W, Yang Y, Liu ZH, Zhao YJ, Zhang Q, Zhang L, et al. Progression of Mental Health Services during the COVID-19 Outbreak in China. Int J Biol Sci. 2020;16(10):1732-8. doi: 10.7150/ijbs.45120. [PubMed: 32226291]. [PubMed Central: PMC7098037].

8. Ali Noorbala A, Badrfam R, Zandifar A. COVID-19 and Psychosomatic Care in the Largest General and Academic Hospital in Iran. J Iran Med Council. 2021;3(4):216-8. doi: 10.18502/jimc.v3i4.5195.

9. Sheikhmoonesi F, Rezapour M, Azizpour M, Ghafour I. Front-Line Medical Staff Experience About the Novel Psychological Intervention of Brief and Online Balint Group: A Qualitative Study in Iran During the 
COVID-19 Outbreak. Iran J Psychiatry Behav Sci. 2021;15(2). e107962. doi: 10.5812/ijpbs.107962.

10. Rezapour M, Zarghami M, Sheikhmoonesi F. [Psychological Experience and Needs of Front-line Nurses during COVID-19 Outbreak in Iran: A Qualitative Study]. J Maz Univ Med Sci. 2021;31(196):125-35. Persian.

11. Galehdar N, Kamran A, Toulabi T, Heydari H. Exploring nurses' experiences of psychological distress during care of patients with COVID-19: a qualitative study. BMC Psychiatry. 2020;20(1):489. doi:10.1186/s12888020-02898-1. [PubMed: 33023535]. [PubMed Central: PMC7538040].

12. Galehdar N, Toulabi T, Kamran A, Heydari H. Exploring nurses' perception of taking care of patients with coronavirus disease (COVID-19): A qualitative study. Nurs Open. 2021;8(1):171-9. doi: 10.1002/nop2.616. [PubMed: 33318825]. [PubMed Central: PMC7729793].

13. Lai J, Ma S, Wang Y, Cai Z, Hu J, Wei N, et al. Factors Associated With Mental Health Outcomes Among Health Care Workers Exposed to Coronavirus Disease 2019. JAMA Netw Open. 2020;3(3). e203976. doi: 10.1001/jamanetworkopen.2020.3976. [PubMed: 32202646]. [PubMed Central: PMC7090843].

14. Gonzalo RM, Ana RG, Patricia CA, Laura AL, Nathalia GT, Luis C, et al. Short-term emotional impact of COVID-19 pandemic on Spaniard health workers. J Affect Disord. 2021;278:390-4. doi: 10.1016/j.jad.2020.09.079. [PubMed: 33007629]. [PubMed Central: PMC7834676].

15. Liu Y, Long Y, Cheng Y, Guo Q, Yang L, Lin Y, et al. Psychological Impact of the COVID-19 Outbreak on Nurses in China: A Nationwide Survey During the Outbreak. Front Psychiatry. 2020;11:598712. doi: 10.3389/fpsyt.2020.598712. [PubMed: 33362609]. [PubMed Central: PMC7759517].

16. Nasimi M, Ahangari N, Lajevardi V, Mahmoudi H, Ghodsi SZ, Etesami I. Quality of life and mental health status in patients with lichen planopilaris based on Dermatology Life Quality Index and General Health Questionnaire-28 questionnaires. Int $J$ Womens Dermatol. 2020;6(5):399-403. doi: 10.1016/j.ijwd.2020.09.005. [PubMed: 33898707]. [PubMed Central: PMC8060658].

17. Yadollahi M, Razmjooei A, Jamali K, Niakan MH, Ghahramani Z. The relationship between professional quality of life(ProQol) and general health in Rajaee Trauma Hospital staff of Shiraz, Iran. Shiraz E-Med J. 2016;17(9). e39253. doi: 10.17795/semj39253.

18. Venugopal VC, Mohan A, Chennabasappa LK. Status of mental health and its associated factors among the general populace of India during COVID-19 pandemic. Asia Pac Psychiatry. 2020. e12412. doi: 10.111//appy.12412. [PubMed: 32830876]. [PubMed Central: PMC7460994].

19. Sterling M. General Health Questionnaire - 28 (GHQ-28). J Physiother. 2011;57(4):259. doi: 10.1016/S1836-9553(11)70060-1. [PubMed: 22093128].
20. Nourbala AA, Bagheri YS, Mohammad K. [The validation of general health questionnaire-28 as a psychiatric screening tool]. Hakim Res J. 2009;11(4):47-53. Persian.

21. Malakouti SK, Fatollahi P, Mirabzadeh A, Zandi T. Reliability, validity and factor structure of the GHQ-28 used among elderly Iranians. Int Psychogeriatr. 2007;19(4):623-34. doi: 10.1017/S1041610206004522. [PubMed: 17069666].

22. Naeinian MR, Nikazin A, Shairi MR. [Factor structure, validity, and reliability of General Health Questionnaire (GHQ-28) in Iranian adolescents]. Clin Psychol Psychother. 2014;12(1):159-72. Persian.

23. Dehvan F, Kamangar P, Baiezeedy S, Roshani D, Gheshlagh RG. The relationship of mental health with resilience among psychiatric nurses. Nursing Practice Today. 2018;5(4):368-74. doi: 10.18502/npt.v5i4.115.

24. Taghinejad H, Suhrabi Z, Kikhavani S, Jaafarpour M, Azadi A. Occupational Mental Health: A Study of Work-Related Mental Health among Clinical Nurses. J Clin Diagn Res. 2014;8(9):WC01-3. doi: 10.7860/JCDR/2014/8247.4835. [PubMed: 25386506]. [PubMed Central: PMC4225958].

25. Al-Amer R, Malak MZ, Aburumman G, Darwish MM, Nassar MS, Darwish M, et al. Prevalence and Correlates of Psychological Reactions Among Jordanian Nurses During the Coronavirus Disease 2019 Pandemic. ResearchSquare. 2020;Preprint. doi: 10.21203/rs.3.rs-35820/v1.

26. Huang L, Wang Y, Liu J, Ye P, Chen X, Xu H, et al. Factors Influencing Anxiety of Health Care Workers in the Radiology Department with High Exposure Risk to COVID-19. Med Sci Monit. 2020;26. e926008. doi: 10.12659/MSM.926008. [PubMed: 32710536]. [PubMed Central: PMC7401832].

27. Nie A, Su X, Zhang S, Guan W, Li J. Psychological impact of COVID19 outbreak on frontline nurses: A cross-sectional survey study. J Clin Nurs. 2020;29(21-22):4217-26. doi: 10.1111/jocn.15454. [PubMed: 32786150]. [PubMed Central: PMC7436701].

28. Puradollah M, Ghasempour M. Necessity of Attention to Mental Health of the Front Line Nurses against COVID-19: A Forgotten Requirement. IntJ Community Based Nurs Midwifery. 2020;8(3):280-1. doi: 10.30476/IJCBNM.2020.85889.1301. [PubMed: 32656281]. [PubMed Central: PMC7334749].

29. Khamis F, Al Mahyijari N, Al Lawati F, Badahdah AM. The Mental Health of Female Physicians and Nurses in Oman during the COVID19 Pandemic. Oman Med J.2020;35(6). e203. doi:10.5001/omj.2020.103. [PubMed: 33335744]. [PubMed Central: PMC7724055].

30. Di Tella M, Romeo A, Benfante A, Castelli L. Mental health of healthcare workers during the COVID-19 pandemic in Italy.J Eval Clin Pract. 2020;26(6):1583-7. doi: 10.1111/jep.13444. [PubMed: 32710481].

31. Jackson D, Usher K. COVID-19: Resilience and the Nursing Workforce. Investigación en Enfermería. 2020;22. 